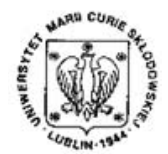

Annales UMCS

Informatica

Lublin-Polonia

Sectio AI

http://www.annales.umcs.lublin.pl/

\title{
The Possibility of Using Artificial Neural Networks for the Estimation of Mass Composition of High-Energy Primary Cosmic Ray
}

\author{
Jan Malinowski $^{1 *}$, Grzegorz Gustek ${ }^{2}$ \\ ${ }^{1}$ Department of High Energy Astrophysics, University of Lodz, \\ Pomorska 149/153, 90-236 Lodz, Poland. \\ ${ }^{2}$ Faculty of Physics and Applied Informatics, University of Lodz, \\ Pomorska 149/153, 90-236 Lodz, Poland.
}

\begin{abstract}
This paper shows that the artificial neural networks (ANN) can be used for determining the type of particles of high-energy primary cosmic ray (i.e. its mass composition) initiating the EAS. The approach implemented here can be used, e.g., in the Auger experiment. We describe the details of the ANN construction and demonstrate that the program is correct and can be further used to solve physical problems. The network was taught and tested based on the data for the maximum of the EAS development (X_max) and primary energy of a particle initiating this EAS $(\lg (\mathrm{E} 0))$. The identification of particles based on $X \_$max and $\lg (\mathrm{E} 0)$ resulted in around $80 \%$ of correct answers for the light mass composition and $99 \%$ for the heavy one. We have a correct answer for the mass composition with domination of one type of particles, i.e. light or heavy. Otherwise, additional parameters should be included as ANN input data.
\end{abstract}

\section{The Objective of this Work}

Registering multiple outcomes of interactions there is a problem how to identify the primary particle type or its other parameters. Since the distributions of registered values are in some areas the same for two types of particles, algorithmic methods cannot be used in this case.

*jmalinowski@uni.lodz.pl 
Artificial neural networks (ANN) have the ability of finding, matching and generalizing similar characteristics. This is why they are sometimes used in physics (see for example [13]). However, in a lot of problems where this tool could be very useful research does not take advantage of it.

In recent years, experiments on EAS initiated by high energy cosmic ray (CR) particles ( around $10^{18}-10^{20} \mathrm{eV}$ ) have been conducted. The experiments Auger and a little older AGASA and Fly's Eye are very well known examples. One of their key objectives is to identify the type of primary particles coming from outer space. In these experiments, detectors register values which have various distributions. For different types of particles, there are areas where their distributions overlap.

This paper presents how ANN can be used to analyze the data registered in detectors and determine the type of primary particles responsible for initiating the registered EAS. The former papers on ANN application to solve physical problems cited above consider the particles registered in different energy regions and rbased on other research areas. Moreover, in paper [4] possible usage of ANN in the Auger experiment is mentioned but the paper does not report the whole analysis.

\section{ANN Construction and Testing}

ANN has neurons located in layers. Each neuron has the activation function. The shape of this function is conclusive for the output value, entire ( 0 or 1$)$ or floating point number $[5,6]$. For solving our problem we used a nonlinear sigmoid activation function of the following form: $y=1 /(\exp (-\beta x))$. The slope coefficient, $\beta$, of the sigmoid is important here since it allows to control the shape of the curve. For any sigmoid arguments values of the function ranges from 0 to 1 what is the most important for network teaching process.

To teach ANN, we used the supervised teaching method, i.e. "with the teacher", and the backward error propagation method. The algorithm of backward error propagation [6] needs a teaching vector on the input and known output data. Teaching means the correction of weights such that errors made by single neurons, and thus by the whole network, decrease in consecutive steps until reaching a satisfactory precision level. The tests of the network were conducted using typical examples like teaching of the AND and XOR functions, identifying 26 letters and reproducing a sinusoid. A satisfactory result of these tests confirmed good work of our implementation.

\section{ANN in Experiment}

The network has to learn the identification of particles initiating the EAS based on the values of X_max received from the simulations. The simulation of EAS is conducted using the CORSIKA program [7].

Our network will detect two types of particles with extreme values of mass number, protons - light particles and iron nuclei - heavy particles. The network is first taught based on the simulation data and then tested based on the computation data. The neural network used in 
this task gives two values, i.e. there are two neurons with the sigmoid function in the output layer. Each of them is supposed to identify one of two types of particles: proton or iron nucleus. Due to the sigmoid activation function, it is possible to identify the similarity of a primary particle to a proton or an iron nucleus.

The data includes X_max (divided by 10), i.e. the depth in the atmosphere where the shower has the maximum number of particles, and $\lg (\mathrm{E} 0)$, where E0 is the energy of the particle initiating EAS. X_max is divided by 10 because its values are much larger than those of $\lg (\mathrm{E} 0)$ and the values given at the input should be of a similar quantity so that too large values alone do not decide about information processing in the network. The data used for teaching the network is presented in Fig. 1. This plot includes 20 different cases of simulation, 10 for protons and 10 for iron nuclei, each of them for different energy. For each case 100 values of X_max have been given. Moreover, Fig. 1 shows that a lot of points overlap which means that for similar data the network would be taught once that it is a proton and once that it is an iron nucleus. Thus the weights of nodes could eliminate one another during sequential iterations of the teaching process. To eliminate a large number of points to learn and still depict an interval of X_max values correctly, we estimated the average $\left.\left(<X \_\max \right\rangle\right)$ and standard deviation $(\sigma)$ of X_max for each of 20 data groups. These data describe an approximative interval of the values of X_max presented in Fig. 2.

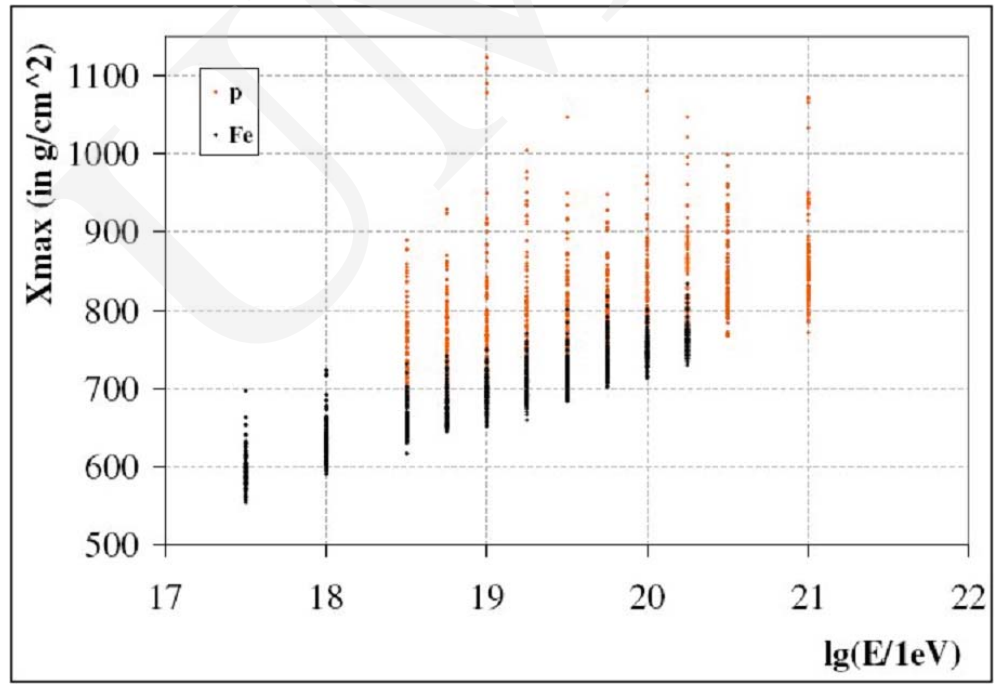

Fig. 1. The values of parameter X_max for EAS depending on the logarithm of the primary particle energy, i.e. the particle initiating EAS.

These approximate values are a sequence for the teaching process. Comparing them with the initial values in Fig. 1, we can observe that they depict the intervals of X_max correctly. There are 60 teaching patterns, 30 for protons and 30 for iron nuclei. Although some patterns still overlap (similar to Fig. 1), their number is very low and they occur in an area where the network should indeed have problems with the identification of particles. To indicate the 
Pobrane z czasopisma Annales AI- Informatica http://ai.annales.umcs.pl

Data: 26/04/2023 11:27:27

126 The Possibility of Using Artificial Neural Networks for the Estimation of ...

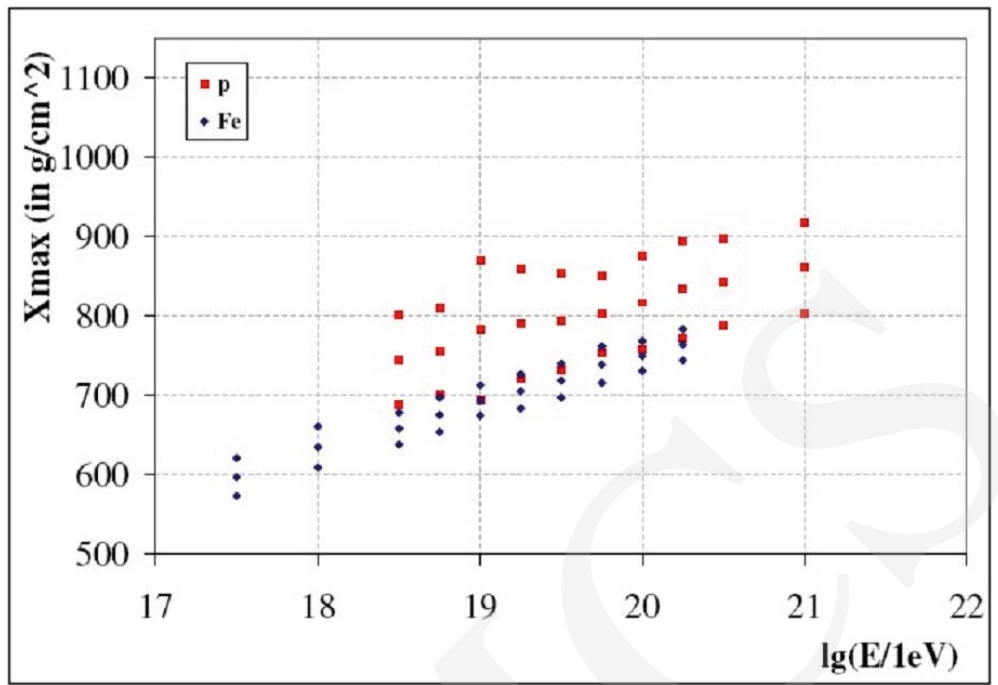

Fig. 2. Points of the teaching sequence $<X \_\max >-\sigma,<X \_\max >,<X \_\max >+\sigma$.

significance of the value $<\mathrm{X} \_\max >$ with respect to $<\mathrm{X} \_$max $>-\sigma$ and $<\mathrm{X} \_\max >+\sigma$, the value $<\mathrm{X} \_$max $>$was given with different weights during the teaching. The results do not depend on the weights of $<$ X_max $>$.

\section{Testing the Network}

Testing of the network consists in drawing a certain number of values X_max, separately for protons and iron nuclei, for each $\lg (\mathrm{E} 0)$ from a normal distribution with the known and previously set means of teaching vectors $<\mathrm{X} \_\max >$ and the known standard deviation $\sigma$ as well as checking how the network identifies the chosen values. In most cases, the network should identify the particle correctly.

Various configurations of ANN have been checked, among others: 1-2, 2-2, 5-2 and 20-2 (the number of neurons in the first and second layers). The following ANN parameters have been analyzed: learning coefficient 0.15 or 0.1 , momentum coefficient 0.4 or 0.3 , weights and thresholds of neurons randomly from the interval $(-0.1,0.1)$ and the slope of sigmoid coefficient 0.1 . Learning of the network was ended when the error decreased to a given value or the desired number of epochs was exceeded.

For ANN with a 1-2 construction, the error after 20000 epochs reached 3.87. Fig. 3a (depicting only 10000 epochs) shows that the learning first evolved unsteadily and there were grate changes of errors. After around 4000 epochs the error stabilized at the level of about 3.88 and then declined slightly. For ANN with a 2-2 construction, the error after 20000 epochs was 3.87 , i.e. its value was similar to the 1-2 network. Fig. $3 \mathrm{~b}$ presents the evolution of the learning process of this network. At first, the process is quite fast but after around 3500 epochs the error starts to fluctuate. The reason for such a result can be too large teaching and 


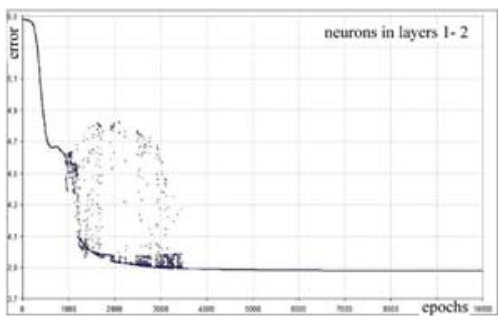

(a)

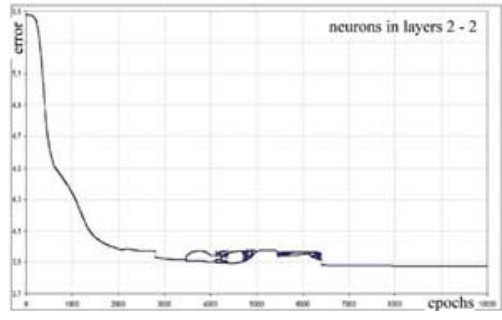

(b)

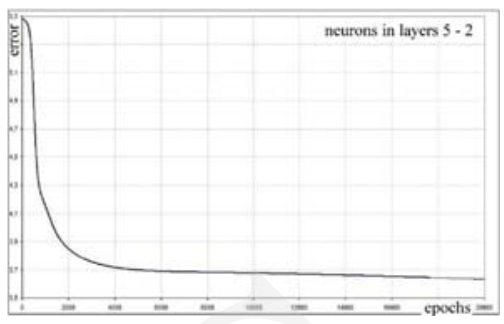

(c)

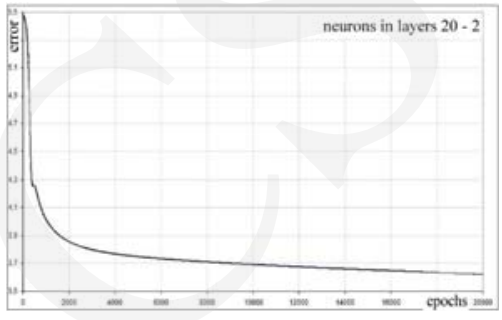

(d)

Fig. 3. Error depending on the number of epochs in the following ANN configurations (from above): 1-2, 2-2, 5-2 and 20-2.

momentum parameters, too small number of neurons and connections among them and the fact that the network is taught to give different answers for similar values. Such cases are visible in Fig. 2 as the points settled in a similar area but correspond to different particles. For a 5-2 network, teaching ended after 20000 epochs with an error of 3.64 equal for all patterns. This value is slightly better than the error values in the former examples. Fig. $3 \mathrm{c}$ shows that the process of learning evolved quite steadily. It seems that this number of neurons is sufficient for teaching the patterns presented above. The 20-2 network gave an error of 3.62 after 20000 epochs. The evolution of the teaching process was similar to the 5-2 network. As shown in Fig. $3 \mathrm{~d}$ a substantially higher number of neurons did not result in a large decrease of the error made by the network.

Consequently, achieving a much better precision during the learning process is impossible for such patterns. The main reason is that they are quite similar for different particles. Too many connections between neurons may sometimes disturb the identification process. Moreover, too many neurons can cause that the network learns patterns very precisely and loses the ability of generalization. For these reasons and due to the testing results, we choose the 5-2 network for further analysis. Fig. 4 presents its scheme.

\section{Identification of Single Particles}

In the second stage of tests, we establish the intervals for the values of outcoming signals for which a particle was classified as light (p) or heavy (Fe). The test involves pooling 1000 X_max for each $\lg (\mathrm{E} 0)$, as described in section 3. Further, the network answers from the interval $(0,1)$ 


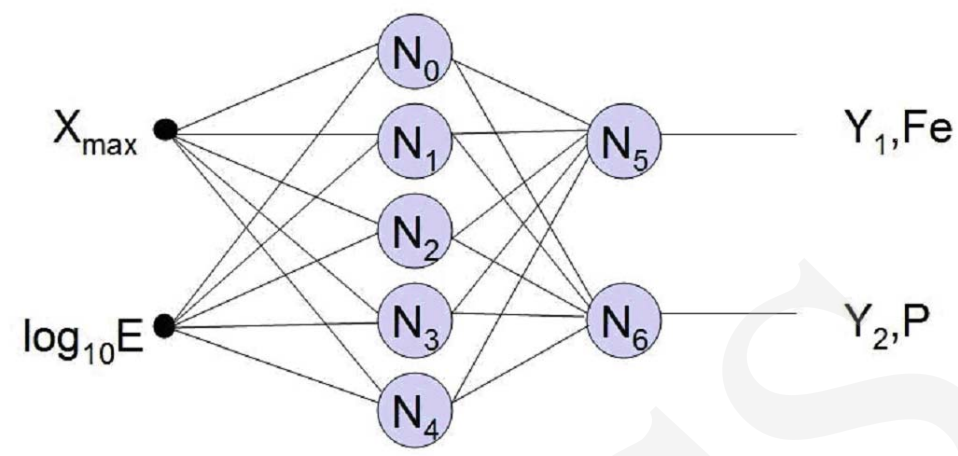

Fig. 4. Scheme of 5-2 ANN used for identification of particles which initiate EAS.

are put to a histogram. After the analysis of histograms, the identification threshold was chosen at 0.7 . The cases with the values from 0.3 to 0.7 are not identifiable, which corresponds to the situation in Fig. 2, where the values of protons and iron nuclei overlap. The values lower than 0.3 mean that the second neuron returns the values higher than 0.7 , i.e. the particle is identified incorrectly.

Table 1. The identification of primary particles type (in \%) for the iron nuclei (cols. 2 and 3) and for the protons (cols. 4 and 5); each sample includes 1000 nuclei.

\begin{tabular}{|c|c|c|c|c|}
\hline $\lg (\mathrm{E} 0 / 1 \mathrm{eV})$ & identified & not identified & identified & not identified \\
\hline 1 & 2 & 3 & 4 & 5 \\
\hline 17.50 & 99.4 & 0.6 & - & - \\
18.00 & 96.0 & 4.0 & - & - \\
18.50 & 97.6 & 2.4 & 56.4 & 43.6 \\
19.00 & 89.7 & 10.3 & 60.7 & 39.3 \\
19.50 & 77.0 & 23.0 & 64.0 & 36.0 \\
20.00 & 61.0 & 39.0 & 65.3 & 34.7 \\
20.50 & - & - & 69.2 & 30.8 \\
21.00 & - & - & 67.8 & 32.2 \\
\hline
\end{tabular}

Table 1, columns 2 and 3, presents the result of identification for the iron nuclei. It shows that for the threshold equal to 0.7 , the identification by this network is very good for some energies, especially lower ones. For higher energies, the number of correctly identified particles decreases to around $60 \%$. However, taking former observations into account, i.e. that the value interval of X_max for both types of particles overlaps, we can expect such identification efficiency. Table 1 , columns 4 and 5, presents the results of identification for protons. It can be seen that the results of tests for protons are slightly worse. The identification rate for all energies is at the level of $60 \%-70 \%$, which is an acceptable result. However, $15 \%$ - $20 \%$ of incorrect identification is not satisfactory. The reason is, as in the case of iron nuclei, 
the same data values for some protons and iron nuclei (see Fig. 1 and 2). Such performance of the network can be thus expected.

Table 2. Simulation parameters.

\begin{tabular}{|c|c|c|}
\hline Nuclei (A) & $<$ X_max $>$ & $\sigma$ \\
\hline $\mathrm{p} \mathrm{(1)}$ & 793 & 61 \\
$\mathrm{He}(4)$ & 765 & 47 \\
$\mathrm{~N}(14)$ & 741 & 35 \\
$\mathrm{Si}(28)$ & 728 & 28 \\
$\mathrm{Fe}(56)$ & 714 & 21 \\
\hline
\end{tabular}

\section{Identification of Particle Groups}

For teaching the network, the same data as in the last case was used. ANN has three inputs The following values are given to them:

$\lg (\mathrm{E} 0)$ - logarithm of primary energy;

$<$ X_max $>$ - average value of X_max;

S_Xmax - standard deviation.

Based on the experience from the tasks described above, we set the ANN architecture to 4-2: 4 neurons in the first layer and two neurons in the output layer, one for detection of the light composition and the other one for the heavy composition.

The network was taught with the parameters as below:

$\mathrm{u}=0.3$ - learning coefficient;

$\mu=0.6$ - momentum coefficient;

$\beta=0.1$ - sigmoid slope coefficient;

$(-0.25,0.25)$ - starting weights, random from the interval;

0.05 - learning accuracy.

The learning process was completed after 32597 epochs.

Different and extremal cases of the cosmic ray mass composition have been assumed for the analysis. Table 3 presents the percentage of nuclei for each case. The test was conducted for the particles with energy 1019.5. Protons, He, N, Si and Fe nuclei have been taken into account. For the calculation of $<$ X_max $>$ for these particles the following fit was taken:

$$
<X \_\max >=(1-B) X o(\log (E / \varepsilon)-\log (A)),
$$

where $\mathrm{Xo}=37 \mathrm{~g} / \mathrm{cm}^{2}, \varepsilon=81 \mathrm{MeV}, \mathrm{B}=0.47, \mathrm{~A}$ - particle mass number.

The standard deviation of X_max distributions was fitted of $\sigma$ for $\mathrm{p}$ and Fe nuclei simulation data. For all tests 1000 values of X_max were drawn from the normal distribution with the parameters presented in Table 2.

Table 3 presents the weighted averages $<\mathrm{X} \_\max >$ and standard deviations $\sigma$ for each model of mass composition. Table 4 shows that ANN identified 3 of 5 cases correctly. Clearly, neural 
network works better when one type of particles dominates. In the case of an equal number of light and heavy particles, ANN interprets them as protons. The reason is that the X_max distributions for protons have larger standard deviations than those for Fe. That is why protons are dominating in the case of partial overlapping of $\mathrm{X} \_$max distributions coming from $\mathrm{p}$ and $\mathrm{Fe}$.

Table 3. Percentage of nuclei in mass composition; average and standard deviation of X_max for models.

\begin{tabular}{|c|c|c|c|c|c|c|c|}
\hline Model & $\mathrm{p}$ & $\mathrm{He}$ & $\mathrm{N}$ & $\mathrm{Si}$ & $\mathrm{Fe}$ & $\left\langle\mathrm{X} \_m a x\right.$ & $\sigma$ \\
\hline 1 & 70.0 & 10.0 & 5.0 & 7.5 & 7.5 & 775.6 & 56.9 \\
2 & 7.5 & 7.5 & 5.0 & 10.0 & 70.0 & 726.7 & 37.4 \\
3 & 5.0 & 5.0 & 80.0 & 5.0 & 5.0 & 743.3 & 38.6 \\
4 & 45.0 & 5.0 & 0.0 & 5.0 & 45.0 & 753.6 & 59.8 \\
5 & 20.0 & 20.0 & 20.0 & 20.0 & 20.0 & 745.4 & 47.2 \\
\hline
\end{tabular}

Table 4. The ANN answers for the assumed models (Answ-Fe - identified by ANN as heavy; Answ-p - identified as light).

\begin{tabular}{|c|c|c|c|}
\hline Model & Answ-Fe & Answ-p & Interpretation \\
\hline 1 & 0.009 & 0.991 & Light \\
2 & 0.823 & 0.177 & Heavy \\
3 & 0.489 & 0.511 & not identified \\
4 & 0.010 & 0.990 & Light \\
5 & 0.038 & 0.962 & Light \\
\hline
\end{tabular}

\section{Conclusion}

The results show that ANN gives correct answers by the detection of the primary cosmic ray mass composition when the particles of one type, i.e. light or heavy, are dominating $(82 \%-$ 99\%). The correct answer, which is the lack of detection, is received also for the composition with the majority of nuclei from the Middle group, i.e. between $\mathrm{p}$ and Fe. Therefore, ANN presented in this paper can be used when the primary cosmic ray includes a majority of only one type of particles, i.e. mass composition is heavy, light or medium.

For the cases when the number of all types of particles in the mass composition is the same, ANN (described in this paper) is not able of giving the correct answer.

In such a case, an additional parameter should be included as ANN input data. Such a parameter should characterize EAS as a function of the type of primary particle. 


\section{References}

[1] Scrimaglio R. et al., Analysis of sileye-3/alteino data with a neural network technique: particle discrimination and energy reconstruction, Advances in Space Research 37 (2006): 1697-1703.

[2] Bussino S., Mari S. M., Gamma-hadron discrimination in extensive air showers using a neural network, Astroparticle Physics 15(1) (2001): 65-77.

[3] Tamada M., Neural net classification of proton- and heavy nucleus-induced cosmic ray families, J. Phys. G: Nucl. Part. Phys. 23 (1997): 497-506.

[4] Tanco M. G., Sciutto S., Tiba A., Neural networks as a statistic diagnostic tool for mass composition at the highest energies, Proc. of the 28th ICRC (Tsukuba, 2003): 587.

[5] Rutkowski L., Metody i Techniki Sztucznej Inteligencji (PWN, Warszawa, 2006).

[6] Duch W., Korbicz J., Rutkowski L., Tadeusiewicz R., Sieci Neuronowe (Exit, Warszawa, 2000).

[7] D. Heck et al., FZKA 6019 (Forschungszentrum Karlsruhe, 1998). 